\title{
Rapid Spectrotemporal Plasticity in Primary Auditory Cortex during Behavior
}

\author{
Pingbo Yin, Jonathan B. Fritz, and Shihab A. Shamma \\ Neural Systems Laboratory, Institute for Systems Research, University of Maryland, College Park, Maryland 20742
}

Complex natural and environmental sounds, such as speech and music, convey information along both spectral and temporal dimensions. The cortical representation of such stimuli rapidly adapts when animals become actively engaged in discriminating them. In this study, we examine the nature of these changes using simplified spectrotemporal versions (upward vs downward shifting tone sequences) with domestic ferrets (Mustela putorius). Cortical processing rapidly adapted to enhance the contrast between the two discriminated stimulus categories, by changing spectrotemporal receptive field properties to encode both the spectral and temporal structure of the tone sequences. Furthermore, the valence of the changes was closely linked to the task reward structure: stimuli associated with negative reward became enhanced relative to those associated with positive reward. These task- and-stimulus-related spectrotemporal receptive field changes occurred only in trained animals during, and immediately following, behavior. This plasticity was independently confirmed by parallel changes in a directionality function measured from the responses to the transition of tone sequences during task performance. The results demonstrate that induced patterns of rapid plasticity reflect closely the spectrotemporal structure of the task stimuli, thus extending the functional relevance of rapid task-related plasticity to the perception and learning of natural sounds such speech and animal vocalizations.

Key words: ferrets; primary auditory cortex; tone sequence

\section{Introduction}

When an animal engages in a behavioral task, sensory cortical processing rapidly adapts so as to optimize task performance. This task-related optimization is influenced by context and task properties (task design, reward valence), by animal training history and motivation, and by the specific sensory stimuli involved (Beitel et al., 2003; Fritz et al., 2003, 2005a, b, 2007, 2010; Brown et al., 2004; Blake et al., 2006; Polley et al., 2006; Selezneva et al., 2006; David et al., 2012; Guo et al., 2013). Thus, when detecting a target tone against a noisy background, the sensitivity of the receptive fields in primary auditory cortex (A1) rapidly changes at the frequency of the target tone (Fritz et al., 2003, 2010; Atiani et al., 2009). The valence of the change depends on the meaning of the tone; if the tone is an aversive stimulus (negative reward), the change is positive (receptive field is sensitized at the target tone frequency); if the tone is associated with a positive reward, the change is negative (receptive field is suppressed at the target tone frequency) (Fritz et al., 2005a; David et al., 2012). These receptive field changes are not observed in be-

\footnotetext{
Received July 1, 2013; revised Jan. 24, 2014; accepted Feb. 19, 2014.

Author contributions: P.Y., J.B.F., and S.A.S. designed research;P.Y. performed research; P.Y. and S.A.S. analyzed data; P.Y. and S.A.S. wrote the paper.

This work was supported by the National Institutes of Health Grant R01 DC005779. We thank S. David, K. Donaldson, L. Ma, and S. Atiani for help with animal surgeries; and N. Mesgarani and S. David for assistance with data analysis.

The authors declare no competing financial interests.

Correspondence should be addressed to Dr. Pingbo Yin, Neural Systems Laboratory, Institute for Systems Research, University of Maryland, College Park, MD 20742. E-mail: pyin@umd.edu.

DOI:10.1523/JNEUROSCI.2799-13.2014

Copyright $\odot 2014$ the authors $\quad 0270-6474 / 14 / 344396-13 \$ 15.00 / 0$
}

haviorally naive control animals when presented with identical acoustic sequences. Moreover, these changes are initiated in trained animals only when they actually perform the task, and not during passive listening. The changes often persist after the task ends, decaying slowly over time (Fritz et al., 2003).

Our studies of rapid spectrotemporal receptive field (STRF) plasticity during behavior have focused on the spectral dimension by using purely spectral stimuli consisting of pure tones or tone complexes (Fritz et al., 2003, 2005b). These studies have shown that the pattern of receptive field changes matches closely the spectral composition of the stimuli, a principle that we have referred to as the "matched-filter" hypothesis (Fritz et al., 2007; Atiani et al., 2009; Mesgarani et al., 2009; David et al., 2012). Rapid adaptive changes, have also been observed along the temporal dimension when the tasks depended purely on the temporal parameters of the stimuli, such as when discriminating a change in click rates, the duration of a tone, and the amplitude modulations (Bao et al., 2004; Fritz et al., 2005b; Wassenhove and Nagarajan, 2007; Dong et al., 2011; Niwa et al., 2012; Shetake et al., 2012).

However, most of our commonly heard acoustic stimuli, such as speech, music, animal vocalizations, and environmental sounds, usually convey information via changes defined jointly along the spectral and temporal dimensions (Selezneva et al., 2006; Mesgarani et al., 2009, 2010; Patil et al., 2012). This study tested the postulate that complex spectrotemporal stimuli would induce a matching spectrotemporal pattern of rapid plasticity. We tested this hypothesis in ferrets trained to discriminate the direction of a variable frequency two-tone pair (Yin et al., 
2010). These ferrets learned to distinguish the direction of a twotone contour independent of the absolute frequency of the tone components. The neurophysiological results from these ferrets confirm that neuronal STRFs adapt during behavior in a manner consistent with enhanced performance and in a pattern that reflected closely the spectrotemporal structure of the task stimuli. These results extend the functional relevance of rapid taskrelated plasticity to the perception and learning of natural sounds rich in spectrotemporal structure, such as speech and animal vocalizations.

\section{Materials and Methods}

\section{Subjects and experimental apparatus}

All experimental procedures in the present study were in accord with National Institutes of Health policy on experimental animal care and use and were approved by the Institutional Animal Care and Use Committee of the University of Maryland. Behavioral and neurophysiological studies were conducted on four adult female ferrets (Mustela putorius), weighing between 600 and $900 \mathrm{~g}$. Two ferrets were trained on a tone-sequence discrimination task, whereas the other two animals were naive controls. During animal training, behavior was continuously monitored visually by video camera and performance monitored in real time by a customized MATLAB program (MathWorks). The two ferrets in the training group were placed on a water-control protocol on which they were typically trained during one daily session for $5 \mathrm{~d}$ per week and obtained $a d$ libitum water over the weekend. Animals received a minimum amount of $20 \mathrm{ml}$ water per training day, and the additional water was supplemented if the animal did not obtain the minimum amount water in performing the task.

Ferrets were trained and tested in a customized-design wire mesh training cage $(20 \mathrm{~cm}$ width $\times 38 \mathrm{~cm}$ depth $\times 23 \mathrm{~cm}$ high), which was placed within a single-walled, sound-attenuated chamber (IAC). A licksensitive waterspout $(2.5 \mathrm{~cm} \times 3.7 \mathrm{~cm})$ stood $12.5 \mathrm{~cm}$ above the floor of the training cage and was connected to a computer-controlled water dispenser (Crist Instrument). A loudspeaker (Primus 162, Infinity) was positioned $40 \mathrm{~cm}$ in front of the cage for sound delivery during behavioral training.

\section{Tone-sequence discrimination task}

The task design and behavioral training procedure were the same as in our previous study (for further details, see Yin et al., 2010). In brief, each trial was initiated when the animal refrained from licking the waterspout for a minimum of $0.5 \mathrm{~s}$. In each trial, a random number (1-5) of reference tone sequences (nontarget) sharing the same frequency contour (either Step-Up or Step-Down for a given animal) was presented, followed by a target tone sequence with the opposite frequency contour. The animal was rewarded with a drop of water when it licked the spout within a given time window after the target sound was presented. The animal received a variable 3-6s timeout penalty if it did not lick the spout during the target sounds. The interstimulus interval (ISI) between all reference and target tone-pair contours was $1.25 \mathrm{~s}$. To discourage licking to the spout during presentation of the reference stimuli, the rewarded drop volume was inversely proportional to the number of false alarms (licking in the time window after each reference sound) of the trial. A training session ended when the animal was satiated (i.e., did not lick the spout in three consecutive trials).

Two domestic ferrets were trained on complementary versions of a Go/NoGo tone-sequence discrimination task. One was trained to detect the upward tone sequence as a target. Thus, this ferret was trained to discriminate the upward tone sequences (the target sequence) from the downward tone sequences (the reference sequence) (Fig. 1A, FerUP). The other behavioral ferret was trained to discriminate downward tone sequences (the target sequence) from the upward tone sequences (the reference sequence) (Fig. 1B, FerDN). The target sound in this paradigm was always appetitive (associated with a positive liquid reward with correct approach), whereas the reference sound was aversive (associated with a negative reward (timeout) if the animal failed to avoid touching the spout). In each tone sequence, the frequency of the tone in the se-
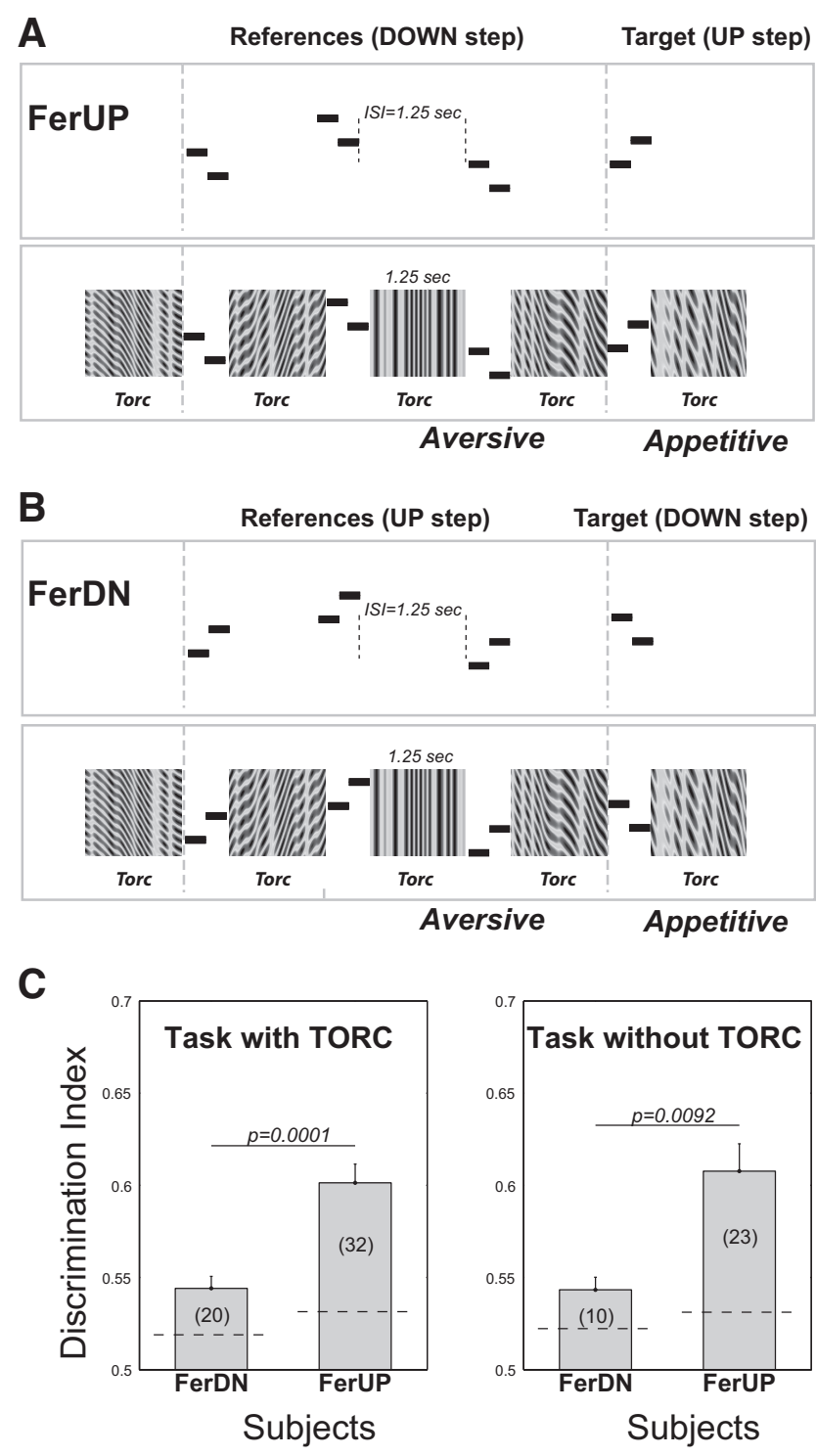

Figure 1. Structure of the behavioral tasks in two animals. $A$, Structure of the trials in ferret FerUP. The animal was trained to listen to acoustic stimuli and to withhold licking during a sequence of tone pairs with downward shifts (reference stimuli: random number of 1-5 repeats). The animal learned to approach the waterspout upon hearing an upward shifting tone pair (target) to receive a water reward. The frequencies of the tone pairs were variable, both within and between trials, and randomly shifted in frequency. In some experiments, only the tone pairs were presented (with silence between them). In others, additional spectrotemporally modulated sounds (such as TORCs or amplitude-modulated rippled noise; see Materials and Methods) were inserted between all tone pairs, and the animals learned to ignore the TORCs and continued to perform the task based on the tone pairs. $\boldsymbol{B}$, Structure of the trials in ferret FerDN. This animal learned the same task as FerUP, except for attending to the opposite tonepair shifts. All other details were the same. $\boldsymbol{C}$, Behavioral performance in head-holder. The bar plot represented the average DI when the animal performed the task with its head restrained. The dashed horizontal lines indicate the threshold level defined as the mean +2 SD of the shuffled DIs (for more details, see Yin et al., 2010). There was no difference in performance between the experimental conditions with and without the TORCs. Although both ferrets displayed significant performance, FerUP had overall a better performance in the head-holder than FerDN (Wilcoxon rank sum test, $p<0.01$ ).

quence was randomized over a 4-octave frequency range; consequently, the animals could not use the absolute frequency to solve the task. Instead, ferrets had to integrate the first tone (T1) with the second tone (T2) together and use the relationship between the two tones to determine pitch direction. Eventually, after several months of shaped training (Yin et al., 2010), the animals learned to attend to relative pitch change regard- 
less of the absolute frequencies of the tone sequences to solve the task. Additional details regarding behavioral training and performance measurements can be found in Yin et al. (2010).

Behavioral assessment. Behavioral performance was assessed by an analysis based on signal detection theory, in which both response accuracy (licks to the water spout after stimulus onset) and the response latency (lick latency from stimulus onset) were used in the determination of a receiver-operating characteristic. The area under the receiveroperating characteristic curve was defined as the discrimination index (DI) for quantifying task performance. DI had a value from 0.5 (for a random performance) to 1.0 (for perfect performance). The learning criterion was defined as a significant DI achieved in three consecutive training sessions (Yin et al., 2010).

Behavioral performance in the head-holder. Two ferrets completed three training phases (Yin et al., 2010) and reached the final behavioral stage with DI between $0.7-0.8$ in the free-running sessions. When behaving under head-restrained conditions, the DIs for both animals declined but remained significantly above threshold levels (as indicated in Fig. 1C, dashed horizontal line). Threshold was defined as the mean plus 2 SD of the shuffled DIs (for more details, see Yin et al., 2010). The decreased DIs when performing in the holder may have been the result of changes in the motor actions during the response. In the free-running condition, the animal typically reacts with its whole body (moving body as well as head and tongue), whereas in the head-fixed condition it can only perform a tongue lick, which some animals find more difficult. There was no difference in performance between the task conditions with or without a temporally orthogonal ripple combination (TORC) background (Fig. 1C). However, better DI scores were obtained with FerUP than from FerDN when performing in the holder (Wilcoxon rank sum test, $p<$ 0.01 ). We note later that this difference apparently correlates with the amount of persistent plasticity after task behavior in the two animals.

\section{Surgeries and physiological recording}

After reaching behavioral criterion (for details of multiple stages of behavioral training, see Yin et al., 2010), a stainless steel headpost was surgically implanted on the ferret skull under aseptic conditions while the animals were deeply anesthetized with $1-2 \%$ isoflurane. After recovery from surgery, the behavioral animals were habituated to a customized head-fixed holder and then retrained on the head-restrained version of the task. The naive control animals also received the same headpost implant and habituation training to the head-fixed restraint holder.

During the implant surgery, the skull covering the auditory cortex was exposed and covered by a thin layer of Zimmer bone cement $(\sim 1 \mathrm{~mm}$ in thickness) and surrounded by a thicker wall built with bone cement $(\sim 3$ $\mathrm{mm}$ in thickness). This formed a bone cement well $\sim 10 \mathrm{~mm}$ in diameter. Subsequently, a small craniotomy $\sim 1 \mathrm{~mm}$ in diameter was made in the center of the well, above primary auditory cortex, to allow access to the underlying brain tissue by microelectrodes for neurophysiological recording (in an initial procedure similar to that of Lu et al., 2001, in the marmoset monkey). The craniotomy was made by drilling through the thin layer of dental cement and $\sim 80-90 \%$ of the thickness of the bone (before reaching the dura) in a target site using a dental drill with a fine drill bit. The remaining bone covering the dura surface was carefully removed using sterilized handheld fine instruments under a Zeiss operating microscope at $25 \times$ magnification to ensure accuracy and completeness of bone removal. The dura was not damaged during this procedure. Because the ferret's skull is thin $(\sim 1.0 \mathrm{~mm}$ in thickness $)$, it was relatively easy to make an opening quickly with little or no disturbance to the animal that was awake during the procedure. A second experimenter was also present to monitor the condition of the animal during the craniotomy procedure, and the animals never showed any visible reaction, response, or distress during the entire procedure. At the beginning and end of each recording session, the craniotomy was thoroughly rinsed with sterile saline. At the end of a recording session, the craniotomy and well was filled with topical antibiotics (in solution) that were rotated on a weekly basis (either Baytril or Cefazolin). After $\sim 10$ min of antibiotic in the whole well, most of the antibiotic was removed, leaving just a small residue sufficient to cover the craniotomy. The area containing the hole was then filled with a silicone ear impression material
(Gold Velvet II-Part Silicon Impression System; Mold Laboratories) that provided a tight seal in the well, thus protecting the craniotomy while the animals were returned to their home cages. The silicone plug could be removed easily at the next recording session. Necessary steps were taken to ensure the sterility during all procedures. After recordings were completed in the original craniotomy, it was gradually enlarged by removing adjacent $\sim 0.5 \mathrm{~mm}$ bone sections over successive months of recording to eventually cover all of A1 in a craniotomy $\sim 3.5 \mathrm{~mm}$ in diameter. The procedure for enlarging the craniotomy was identical to that followed for drilling the original craniotomy. The enlarged craniotomy allowed for recording from the entire primary auditory cortex, and we found we were able to stably hold neurons for many hours during recording in the larger craniotomy.

Recordings were conducted using tungsten microelectrodes $(2-5 \mathrm{~m} \Omega$, FHC) introduced through the craniotomies and controlled by independently moveable drives (Electrode Positioning System, Alpha-Omega). Raw neural activity traces were amplified, filtered, and digitally acquired by a data acquisition system (AlphaLab, Alpha-Omega). Multiunit neuronal responses were monitored online (including all spikes that rose above a threshold level of 4 SDs of baseline noise). Single units were isolated off-line by customized spike-sorting software, which was based on a PCA and template-matching algorithm (Meska-PCA, NSL).

\section{Acoustic stimuli and experimental configuration}

A dense set of individual tone-pips was used to characterize the tuning properties of the neurons. The tone-pips were $150 \mathrm{~ms}$ in duration, spanned a 5-7 octave range with one-fourth octave increments, and were presented at multiple intensity levels (25-75 dB SPL). The characteristic frequency or best response frequency $(\mathrm{BF})$ were computed online from the evoked multiunit activity. This tuning information was used to guide the choice of stimuli presented in a given session to maximize neural responsiveness.

Neurons were also characterized by a set of broadband-modulated noise stimuli called TORCs (Klein et al., 2000). The TORCs were $1.25 \mathrm{~s}$ in duration and were presented passively in isolation or as nonrelevant "background" sounds (Fig. 1A, bottom) during the tone-sequence discrimination task. The TORCs were noise-like in character and provided no pitch-relevant information to the animals, and hence did not convey specific pitch shift percepts to the ferrets that would be helpful for task performance. The animals learned to ignore these task-irrelevant stimuli (all TORC stimuli were behaviorally neutral), focusing instead on discriminating pitch shift direction of the task-relevant tone-pair sequence stimuli. Responses to the TORCs were used to measure the STRFs of the recorded neurons while the animals performed the task (Fritz et al., 2003).

All two-tone sequences used were $300 \mathrm{~ms}$ in duration. Each tone component in the two-tone sequence was an enveloped burst $150 \mathrm{~ms}$ in duration, and there was no silent gap between the two tones in each tone-pair sequence. The frequencies of tone components in the sequences were randomly picked within a 4-octave range around the neurons' BFs, with one-half octave spacing therefore making up a total of 81 $(9 \times 9$ frequencies $)$ two-tone sequences. A subset of these sequences was selected to use in behavioral sessions, which include up to 18 tone sequences (half upward and half downward sequences made up of 9 tone pairs: $(4 \times 2)+1$ and the topmost tone-pair goes up to 4.5 octaves above the lowest) with a fixed one-half octave frequency separation between the two tones in each tone-pair sequence.

Two slightly different sets of stimuli were used for experiments on trained animals during physiological recording: acoustic sequences with and without TORC components. The experiment with TORCs had three blocks that included: (1) pre-behavior passive listening in which TORCs were passively presented in isolation to measure the neuron's STRF; (2) an active listening when the animals performed the tone-sequence discrimination task in which TORCs were used as nonrelevant "background" noise to fill the $1.25 \mathrm{~s}$ ISI gap between the tone sequences, as depicted in Figure 1A, $B$ (bottom panels); and (3) post-behavior passive listening block in which TORCs were again passively presented in isolation. In this set of experiments, the neuron's STRF could be computed from the neural response to TORCs in each of these three blocks, thus 
A $\Delta$ (reference,target) $\sim$ Receptive Field Plasticity
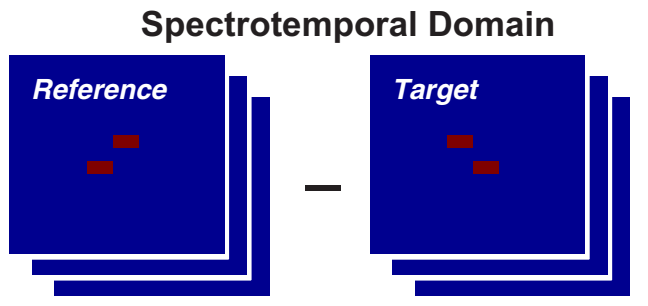

\section{$\sim \Delta S T R F$}

B

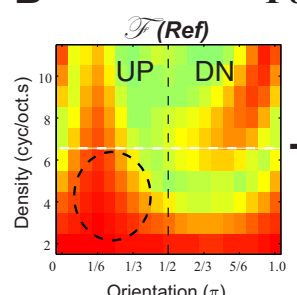

Fourier Domain
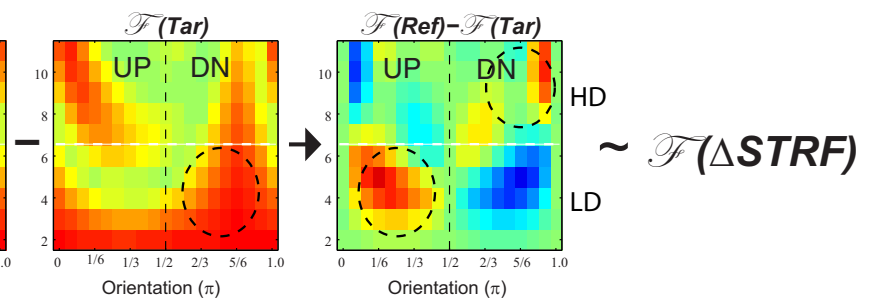

C

Linear arrangement
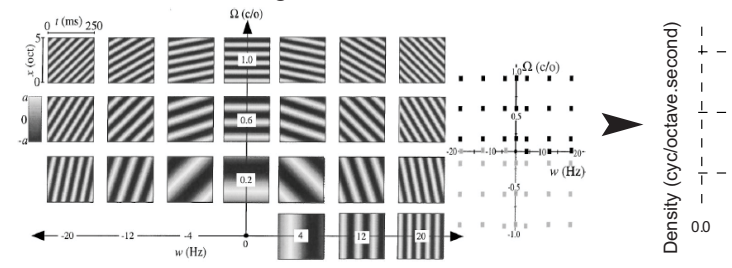

Polar arrangement

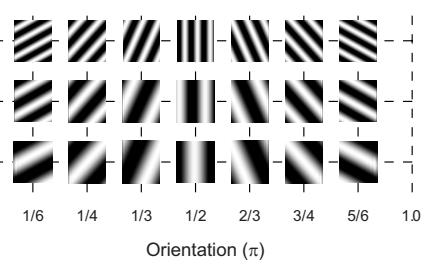

Figure 2. The matched filter hypothesis and polar Fourier representation. $\boldsymbol{A}$, Schematic of the predictions of the matched filter hypothesis. Rapid receptive field plasticity during behavior is predicted to reflect the difference between the discriminated reference and target sounds: $\Delta$ (reference, target). Animals attended to a spectrotemporal difference: the direction of pitch shift in tone pairs that have random absolute frequencies. Consequently, plasticity is predicted to reflect the change in pitch direction via $\triangle S T R F$ of the responding neurons. $\boldsymbol{B}$, The $2 \mathrm{D}$ polar Fourier transforms of the two-tone sequences. The stimuli, STRFs, and $\triangle$ STRFs can be better visualized through their Fourier transforms, which specifically highlight the directional information in the stimuli through their asymmetry around the midline. In general, directionally shifting stimuli or tilted STRFs exhibit an asymmetric Fourier transforms. For the tone pairs, the asymmetry is reversed in the LD and HD regions, which are separated by the white horizontal line in the panels. Because of the "aversive" nature of the reference stimuli (compared with the "appetitive" target), the asymmetry of the Fourier transforms of $\triangle S T R F$ (denoted by $F(\Delta S T R F)$ ) is predicted to match the asymmetry pattern of the Fourier transform of the difference of the reference and target stimuli: $F$ (Ref) $-F(T a r)$. The example shown represents the case when the reference (target) stimulus is the up-shifting (down-shifting) tone sequence. C, All Fourier transforms shown are plotted on a polar grid, which spreads them out more evenly and clearly. The sinusoidal spectra (called "ripples") are the basis functions of the Fourier transform of the spectrotemporal stimulus and STRF patterns. Left panels, Normal distribution of ripple coefficients in the Fourier domain, with positive rates $(\omega)$ and spectral densities $(\Omega)$ corresponding to downward-moving ripples, and negative rates to upward-moving ripples. In the polar Fourier transform, the coefficients of the ripples are simply rearranged on a grid such that all ripples in a given column have the same orientation $(\pi)$ and are organized from 0 to $\pi$ orientations. They are arranged with increasing density up the ordinate.

enabling tracking of changes in the neuron's STRF while the animal engaged in the sequence discrimination task (compared with the passive listening STRF).

In the experimental set without TORC background, the pre-behavior and post-behavior passive listening blocks were not TORCs, but rather the 81 combined two-tone sequences of the 9 frequencies around neuron's the BF. A subset of these 81 tone sequences was used in the block of the sequence discrimination task without the TORC background sounds (Fig. $1 A, B$, top). The directionality functions from the recorded neurons were computed from the neural response to the same tone sequences in all three blocks (i.e., during both pre-behavior/post-behavior passive listening and also during active task performance). The neurophysiological data collected from A1 recordings of the naive animals also included similar datasets from experiments with and without TORC background stimuli (in the ISI in trial stimulus sequences). Thus, we gathered similar types of responses to the tone sequence and TORCs from both the naive animals and the trained animals while they passively listened to the same stimulus sets without behavioral context. In the passive condition, the animals did not lick any stimuli and treated all stimuli as behaviorally neutral. All stimuli used in the experiments were ramped with $5 \mathrm{~ms}$ rise-fall time and presented between 60 and $75 \mathrm{~dB}$ SPL.

\section{Data analysis}

STRF analysis and the modulus of the Fourier transform. STRFs were computed from the neuronal responses to TORCs by using the reverse-correlation method (Klein et al., 2000; Depireux et al., 2001; Miller et al., 2002) during each of the three experimental blocks for the experiment set with TORC background. The variance of the response was estimated using a bootstrap procedure (Miller et al., 2002; Fritz et al., 2003), and the signal-to-noise ratio was computed for each STRF. Neurons were excluded from further analysis if their signal-tonoise ratio $<0.2$ in any of the three measured STRFs.

According to the "matched-filter" hypothesis, receptive field plasticity reflects the difference between reference and target stimuli (Fritz et al., 2007; Mesgarani et al., 2010). Consequently, STRF changes $(\Delta$ STRF) are predicted to mirror the difference between the spectrotemporal patterns of the target and reference tone sequence. Figure $2 A$ schematically illustrates this hypothesis for the case of a DOWN-step target tone sequence.

The moduli of the Fourier transform of the 2D tone sequence spectrograms, denoted by $\mathrm{F}$ (Ref) and F (Tar), are shown in Figure 2B. Each of these plots depicts the weights of the Fourier basis functions that superimpose to make up the patterns (also called ripples, shown in Fig. 2C) (Klein et al., 2000). In these plots, the Fourier coefficients were rearranged on polar coordinates to spread them out and enhance their visual clarity (David et al., 2006). This transformation is explained in Figure 2C. Initially, the coefficients computed for each of the ripple basis functions are arranged as in Fig. 2C (left). To arrange them on a polar plot, the coefficients are simply repositioned such that each column contains those of ripples with the same (spectrotemporal) orientation (the energy sweeps in the spectrogram). The density in polar arrangement is defined by the ripple spectral density (cycle/octave) and its temporal modulation rate (Hz or cycle/second) (i.e., it has units of cycle/octave.s). Horizontally, the coefficients are arranged with increasing orientation (from 0 to $\pi$ ), as shown in Fig. $2 C$ (right). Along the ordinate, the ripples increase their density (while maintaining their orientation).

The key feature of interest in our experiments is the STRF sensitivity to the direction of the tone sequence contour. This directionality is reflected in the asymmetry of the Fourier transform plots around the midline (Depireux et al., 2001). Specifically, when the tone sequence shifts upward (Fig. $2 B$, left), the F (Ref) is asymmetric about the vertical midline with upward (UP) $>$ downward (DN) in the lower densities (LDs, the region includes the densities from 2 to 6 cycles/octave.s, i.e., below the dashed white line); it is asymmetric in the opposite direction (DN $>$ UP) in the higher density region (HD, which includes the densities from 7 to 11 cycles/octave.s, i.e., above the dashed white line). This distinctive "reversed" pattern of asymmetry in HD and LD regions is the result of the 
spectrotemporal nature of the tone pair. The specific location of the reversal (or the horizontal border between the LD and HD regions) depends on the parameters of the tone sequences (duration, step-size, temporal interval of the tone components). If the tones are shorter, or the step between them is larger, the horizontal line would shift up; the opposite happens when the tones are longer or the steps smaller.

When the Fourier transforms are subtracted ( $\mathrm{F}(\mathrm{Ref})-\mathrm{F}(\mathrm{Tar}))$, the resulting plot is highly asymmetric (Fig. 2B, rightmost panel) with the asymmetry of the LD and HD regions clearly reversed above and below the dashed horizontal line. Therefore, the asymmetry of all plots in this paper will be reported separately for these two regions. To quantify the asymmetry of the Fourier transforms, we defined an asymmetry index $\left(\Delta_{\text {sym }}\right)$ as follows:

$$
\begin{aligned}
& \Delta_{\text {sym }}=\left(\mathrm{P}_{\mathrm{UP}}>\mathrm{DN}-\mathrm{P}_{\mathrm{DN}}>\mathrm{UP}\right) / \\
& \left(\mathrm{P}_{\mathrm{UP}}>\mathrm{DN}+\mathrm{P}_{\mathrm{DN}}>\mathrm{UP}\right),
\end{aligned}
$$

where $\mathrm{P}_{\mathrm{UP}>\mathrm{DN}}$ denoted the total power difference between the pixels whose values in the UP quadrant were greater than the corresponding pixels in the DN quadrant, and $\mathrm{P}_{\mathrm{UP}<\mathrm{DN}}$ denoted the analogous total power difference for the opposite pixels. The $\Delta_{\text {sym }}$ ranged from -1 (biased to DN) to 1 (dominated by UP). It was calculated separately for the LD and HD regions of the Fourier transforms.

Basic properties of the STRFs were also measured, such as their BFs, bandwidth, and separability (Depireux et al., 2001; Shechter and Depireux, 2007) and are commented on in Results. A key property of interest here is the upward/downward selectivity of the STRF, which is reflected in the upward or downward tilt of STRF shapes. As in the case of the stimuli, the directionality can be captured by the asymmetry of the Fourier domain, F (STRF), illustrated in Figure $3 A$ (UP-selective neuron) and Figure $3 B$ (DN-selective neuron). To depict the orientation selectivity of the STRF correctly, it is necessary that each STRF or $\triangle$ STRF be left-right reversed before transforming it because a downward (upward) tilted STRF is indeed more selectively responsive to up (down) oriented ripples (Klein et al., 2000; Depireux et al., 2001).

Therefore, in all figures and text, we use the notation " $F($.$) " to$ denote the modulus of the Fourier transform for stimuli and STRFs from neural responses. However, it is important to remember that all transformed neural measures, $F(S T R F)$ and $F(\Delta S T R F)$, include a reversal of the STRFs before performing the Fourier transform. This reversal is not necessary for the stimuli $\mathrm{F}$ (Ref) and $\mathrm{F}$ (Tar). For display purposes only, some regions in the $\mathrm{F}($.) plots will be highlighted by dashed circles to indicate that they were predicted to be enhanced compared with the corresponding regions across the midline as shown in Figure $2 B$.

The computational procedures used to evaluate the STRF changes that occur during task performance are illustrated in Figure 3C. The STRF is measured during the passive state before behavior $\left(\mathrm{STRF}_{\mathrm{Pre}}\right)$ and then during task performance $\left(\mathrm{STRF}_{\mathrm{Dur}}\right)$. The difference between the two measurements $\left(\triangle S T R F_{\text {Dur-pre }}\right)$ is then Fourier transformed to $\mathrm{F}\left(\Delta S T R F_{\text {Dur-pre }}\right)$. The $\mathrm{F}\left(\Delta \mathrm{STRF}_{\text {Dur-pre }}\right)$ obtained from each neuron is normalized to its overall median by its SD. Because these F (.) plots are invariant to frequency translations of the stimuli relative to the BFs, it was possible to accumulate data from many cells by computing the average of all measurements made in each animal (FerUP, FerDN, and naive) and under all three different conditions. The overall $\mathrm{F}(\triangle \mathrm{STRF})$ from the neuron populations in the trained FerUP and FerDN, and naive TarUP and TarDN, were represented by the population median. To reduce the noise, we reserved only the pixels in the population median that were significantly different from zero. To do so, we performed a rank test at each pixel across all the neurons, and significance was only accepted if the pixel and at least one of its neighbors had a $p<0.01$.

Directionality analysis for responses to pure tone sequences. For the experimental data using tone sequence stimuli only (i.e., without intervening TORCs, as in Fig. $1 A, B$, top), the frequency response area for each neuron was derived during passive listening before task performance. The neuron's BF was determined by the onset response evoked by the first tone (T1) in the tone sequences. The preference of the response to the direction of the tone sequences was defined as a directional index $\left(\Delta_{\mathrm{DIR}}\right)$ and computed by the onset responses to the second tone (T2):

$$
\Delta_{\mathrm{DIR}}=\left(\mathrm{R}_{\mathrm{UP}}-\mathrm{R}_{\mathrm{DN}}\right) /\left(\mathrm{R}_{\mathrm{UP}}+\mathrm{R}_{\mathrm{DN}}\right) \text {, }
$$

where $\mathrm{R}_{\mathrm{UP}}$ and $\mathrm{R}_{\mathrm{DN}}$ are the onset responses to $\mathrm{T} 2$ (25-75 ms period after T2 onset) during a two-tone UP or DN step, respectively. The positive index indicates a larger response evoked by T2 during an UP step than during a DOWN step, and vice versa. The $\Delta_{\mathrm{DIR}}$ was computed at the same T2 frequencies for all three experimental blocks (pre-task passive, during behavioral task, post-task passive conditions). The functions of $\Delta_{\text {DIR }}$ versus T2 frequency were obtained for pre-task, during task, and posttask conditions for all neurons. The averaged population functions of $\Delta_{\text {DIR }}$ versus T2 frequency were aligned to the neurons' BFs, and T2 frequency was limited to a range within 0.5 octaves around the BF. The significance of the changes of $\Delta_{\mathrm{DIR}}$ during the task was determined by a paired Wilcoxon signed rank test $(p<0.05)$ within the populations, and by a Wilcoxon rank sum test $(p<0.05)$ between the neuron populations (FerUP vs FerDN for the behavior group; TarUP vs TarDN for the naive group).

\section{Predicting the valence of rapid plasticity}

We have proposed that rapid plasticity is a mechanism to optimize perception by enhancing the brain's ability to distinguish between sensory targets of interest (foreground) versus reference (background) stimuli. According to this hypothesis, the form of receptive field plasticity in a given auditory task context is shaped by the spectrotemporal difference between target and reference stimuli. However, the sign of the plasticity change (i.e., which of the two stimuli is enhanced or suppressed) is influenced by other non-stimulus-related factors, such as task reward valence. Based on previous findings (Fritz et al., 2005a; David et al., 2012), we predicted that $\Delta$ STRFs reflected enhanced responses to aversive stimuli (that the animal seeks to 
avoid) and suppressed responses to appetitive stimuli (that the animal seeks to approach).

In all experiments here, the reference stimuli were always aversive (associated with avoidance of negative feedback [combination of timeout and reward volume reduction] that the animals received if they touched the waterspout), whereas the target stimuli were appetitive (positive reward with approach to the spout). Consequently, we predicted that the $\triangle$ STRFs for the two trained animals in this study (FerUP and FerDN) would change in opposite ways because of the different meanings of the stimuli (Fig. $1 A, B$ ). Specifically, we predicted that the asymmetry of $F(\triangle S T R F)$ would match that of $F$ (Ref), the Fourier transform of the corresponding reference tone sequences. Thus, STRF changes in FerDN should resemble those in Figure $2 B$ (rightmost panel): there would be a preference for the UP side in the lower densities $(\mathrm{LD}>0)$ and the reverse at high densities $(\mathrm{HD}<0)$. The opposite plasticity patterns were predicted for FerUP. However, the asymmetry of the F ( $\Delta$ STRF) was expected to be generally more reliable in the LD range because most STRFs in ferret A1 have their best responses in that range (Kowalski et al., 1996).

\section{Animal numbers and experimental conditions}

A total of four animals were used: two trained and two naive ferrets. The tasks were difficult for the ferrets to learn and took several months for each of the animals to master to behavioral criterion (Yin et al., 2010). Rather than training both animals on the same task version, our approach was to train the two animals on opposite versions of the tasks to act as controls for each other. According to our hypothesis, the predicted patterns of change should go in opposite directions for ferrets trained on opposite task versions. This approach, and combination of opposite training procedures for the two ferrets, provided the best test of our hypothesis because the predictions were so clear and falsifiable. More importantly, the predicted patterns themselves were not simple, but rather highly unintuitive, having opposite asymmetries in the different density regions of the Fourier transforms as discussed in Figure $2 B$.

To further support these interpretations, we considered multiple additional controls: (1) we added two naive animals to the experiments so as to demonstrate that none of the myriad changes in tone sequence responses or STRFs was observed in naive animals; and (2) we developed a new approach to demonstrate spectrotemporal plasticity using a completely different response measure that was derived from the responses to the pure tone sequences, rather than to the TORCs (as in the calculation of the STRFs). We found that the results from the changes of STRFs tended to match the conclusions from the responses to the tone sequences. Moreover, in conducting these new analyses for directionality, we recorded from completely separate neuronal populations from the two trained animals (i.e., distinct from the neuronal populations studied for STRF changes). This allowed us to test whether we could independently confirm the results using different methods in nonoverlapping populations. In each of the controls, we tested and reported the significance of the observed pattern of change, and we did so in the context of the complex predicted patterns of change. For instance, it was highly unlikely that the pairing of LD and HD indices could have by chance displayed the predicted patterns we show later in the paper.

\section{Results}

\section{Summary of the datasets}

Auditory cortical responses to tones in the two-tone contour sequences were usually larger in one direction of the step, as described previously (Shamma and Symmes, 1985; McKenna et al., 1989; Brosch et al., 1999; Wehr and Zador, 2005). However, this directional selectivity depended in a complex way on the frequency of the tone sequences relative to the tuning curve of the neurons as well as the dynamics and spectral shape of the cells' STRFs. Therefore, to demonstrate the presence of rapid receptive field plasticity for this spectrotemporal task, we used alternate approaches to extract stable and invariant response properties based on two methods: Fourier representations of the STRF mea-
Table 1. Summary of the datasets (single units) ${ }^{a}$

\begin{tabular}{|c|c|c|c|c|}
\hline \multirow[b]{2}{*}{ Ferrets } & \multicolumn{2}{|c|}{ No-TORC background } & \multicolumn{2}{|c|}{ TORC background } \\
\hline & TarUP & TarDN & TarUP & TarDN \\
\hline FerUP & 115 & NA & 112 & NA \\
\hline FerDN & NA & 58 & NA & 65 \\
\hline Naive & 77 & 66 & 79 & 63 \\
\hline
\end{tabular}

${ }^{a}$ Two neuronal populations were collected from all animals during the experiments with and without TORC background sounds. TarUP and TarDN indicate stimuli sequences illustrated in Figure $1 \mathrm{~A}$ and Figure $1 \mathrm{~B}$, respectively. NA, No data were collected.

surements (from TORC responses) and directly from responses to the tone sequences.

Thus, the present study included two datasets that were collected independently in each of the four trained and naive animals (Table 1). One dataset was collected when animals were engaged in a pure tone-sequence discrimination task (Fig. $1 A$, top, $B$ ) or when passively listening to the tone sequences with the same sequence context in naive ferrets. This dataset included 115 single units from FerUP (upward tone sequence target) and 58 single units from FerDN (downward tone sequence target). The control data were 77 and 66 single units from two naive ferrets while passively listening to the similar tone sequences TarUP (corresponding to FerUP) and TarDN (corresponding to FerDN), respectively. The second dataset was collected when animals were engaged in a tone-sequence discrimination task in which the TORCs were played as the background sounds during the ISI (Fig. $1 A$, bottom, $B$ ) or when passively listening (to the same tone sequences with interleaved TORCs) in naive ferrets. From the two behavioral ferrets, this dataset included 112 single units from FerUP and 65 single units from FerDN. From the two naive ferrets, this dataset included 79 single units while passively listening to the sequence TarUP and 63 single units while passively listening to the sequence TarDN. All data from the two naive animals were combined under designation of "naive" for the analysis and displays.

Single units were recorded from the A1 left hemisphere in all ferrets, and also from the right hemisphere of FerUP. In all 4 ferrets, units' BFs covered most of the tonotopic region of the A1. They ranged between 0.2 and $14.0 \mathrm{kHz}$ in FerUP, 0.3 and $13.5 \mathrm{kHz}$ in FerDN, and 0.2 and $14.0 \mathrm{kHz}$ in the naive ferrets. Unit responses from all task trials were included in the analysis.

\section{STRF plasticity and directional selectivity}

In the dataset with background TORCs, the changes of neuronal response properties between the passive and behaving states were measured by analyzing the changes of the STRFs ( $\Delta$ STRF). These $\Delta S T R F$ s were constructed from the neuronal responses evoked by the TORCs before and during performance of the tonesequence discrimination task. In general, the strength of the changes in the $\triangle$ STRF of a cell depended on the stimulus frequencies relative to the BF of the cell. However, because of the randomly varying tone-pair frequencies relative to the $\mathrm{BF}$, it was impossible to interpret the $\triangle \mathrm{STRF}$ in the usual spectrotemporal domain because the animals' discrimination of up/down contour direction is presumably taking place in a higher (more abstract) feature space than frequency. Another challenging problem was how to combine $\Delta$ STRFs from many cells so as to compute a population average, a procedure that usually requires an alignment of the STRFs relative to the effective stimulus. In the past, stimuli were held constant throughout the experiment (e.g., the target tones in Fritz et al., 2003 and/or reference tones in Fritz et al., 2005b), so it was straightforward to align the STRFs relative to 
those stimuli. In other studies, we had chosen to illustrate task-related receptive field plasticity by aligning the STRFs relative to their own BF rather than to stimulus frequency (Atiani et al., 2009).

In the present case, however, neither of these alignment approaches was suitable for analyzing receptive field plasticity at a population level because both reference and target tone sequences were random in frequency within a trial and throughout the experiment (Yin et al., 2010). Instead, it was necessary to stabilize the representation of the tone sequences and STRFs with respect to frequency translations by transforming them to the modulus of their Fourier transformation, as depicted in Figures 2 and 3.

STRFs changed during each behavioral session by becoming more sensitized to the direction of the similar shifts of the reference tone sequences (always UP or always DOWN steps). The changes occurred in many STRFs regardless of their original directional selectivity, bandwidth, BFs, or other properties. This is illustrated by the four single units in Figure 4. Two A1 neurons from FerUP (Fig. 4A) had initial passive STRFs with opposite tilts, as indicated by the asymmetry of their F $\left(\mathrm{STRF}_{\text {pre }}\right)$. During behavior, both STRFs changed relative to their prior passive states so as to enhance the preference of the STRF sensitivity toward downward shifts (Fig. 4A, rightmost panels). This is reflected by the asymmetries of $\mathrm{F}$ $\left(\Delta \mathrm{STRF}_{\text {Dur-Pre }}\right)$, which exhibit the anticipated opposite changes at $\mathrm{LD}$ and $\mathrm{HD}$ regions $(\mathrm{LD}<0$ and $\mathrm{HD}>$ 0 ), indicating that the cells became more responsive to downward shifting tone sequence during the task. Two other Al cells were isolated in FerDN (Fig. 4B), where one neuron (top) was initially approximately directionally symmetric and the other (bottom) had a strong preference to downward ripples. During behavior, both STRFs became more sensitized to upward shifting tone sequence, exhibiting $\mathrm{F}\left(\Delta \mathrm{STRF}_{\text {Dur-Pre }}\right)$ values with an opposite asymmetry at the $\mathrm{LD}$ regions $(\mathrm{LD}>0)$ and at the $\mathrm{HD}$ region $(\mathrm{HD}<0)$.

Given the expected variability across a population of cells with a wide range of STRF properties, we computed the averaged $F$ $\left(\Delta \mathrm{STRF}_{\text {Dur-Pre }}\right)$ from 112 neurons recorded in FerUP and 65 neurons in FerDN, as shown in Figure 5. In both animals, there was a significant enhancement of the directional sensitivity of the cells during behavior (Fig. 5A) consistent with enhanced detection of the pitch contours in reference tone sequence during the task. The asymmetry is highlighted by comparing each of the LD and HD regions within the dashed circles to the corresponding region on the other side of the midline. For each animal, there were larger enhancements within the predicted region resulting in $\mathrm{LD}<0$ (i.e., $\mathrm{LD}=-0.9$ ) in FerUP (Fig. 5A, top left) whereas $\mathrm{LD}>0$ (i.e., $\mathrm{LD}=0.7$ ) in FerDN (Fig. $5 A$, top right). Also as predicted, the opposite asymmetry occurs in the HD regions with stronger activation in the $\mathrm{UP}(\mathrm{DN})$ regions leading to $\mathrm{HD}>0$ (i.e., $\mathrm{HD}=0.3$ ) in FerUP and $\mathrm{HD}<0$ (i.e., $\mathrm{HD}=-0.3$ ) in
FerUP. These patterns of indices were also examined on an individual cell basis through the scatter plots of $L D$ versus $H D$ indices (Fig. 5A, bottom). The asymmetric indices are significantly shifted toward opposite sides of the diagonal in the two animals. The statistical significance of this pattern is confirmed by the histograms of the difference between the indices (LD - HD) that are overlaid on the plots, showing a significant shift to negative (positive) values in FerUP (FerDN) (paired Wilcoxon signed rank test: $p=0.0389$ in FerUP and $p=0.0004$ in FerDN). To discover whether there was any topographic pattern in the observed tasked-related changes, the neuronal population from each animal was split into two subpopulations according to neuronal BF: low BF $(1000-4000 \mathrm{~Hz})$ and high BF $(4000-16000 \mathrm{~Hz})$. A comparable pattern of the changes was observed in the subpopulations for both FerUP and FerDN; hence, task-related changes were independent of BF.

The enhancement of the representation of the reference tone pairs persisted after task performance in one animal (FerUP), as demonstrated by the asymmetry of the post-behavior $\mathrm{F}$ $\left(\Delta \mathrm{STRF}_{\text {Post-Pre }}\right)$ measured after the behavioral experiment (Fig. $5 B)$. However, the plasticity in the second animal (FerDN) was present only during performance of the behavioral task and did not persist. A possible explanation of this finding could be related to a previous observation that the magnitude of plasticity effects is correlated with behavioral performance. FerUP did indeed perform its tasks at a more proficient level than FerDN (Fig. 1C). 
A
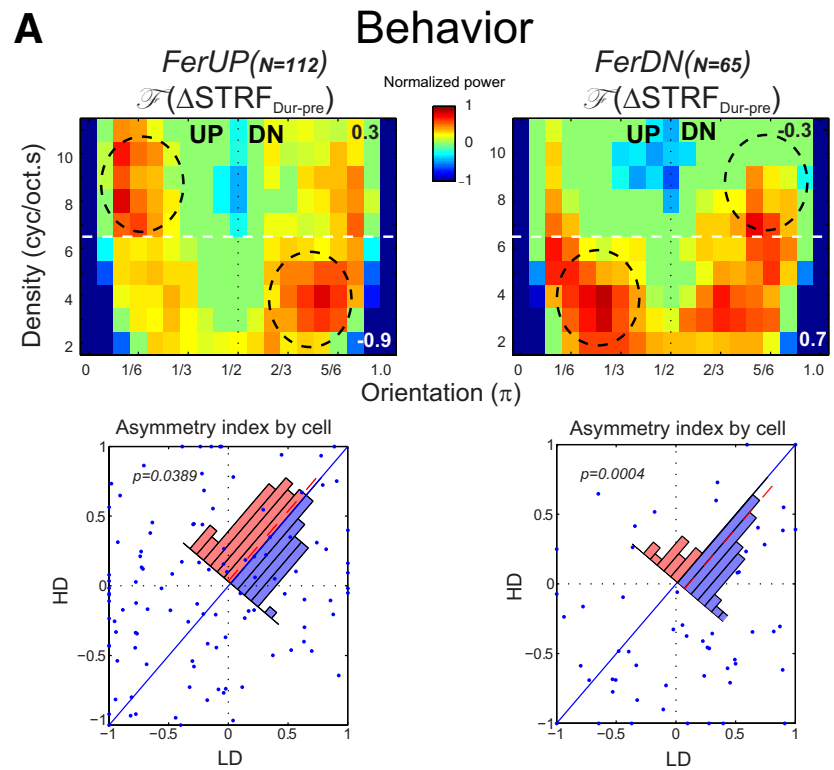

\section{B}
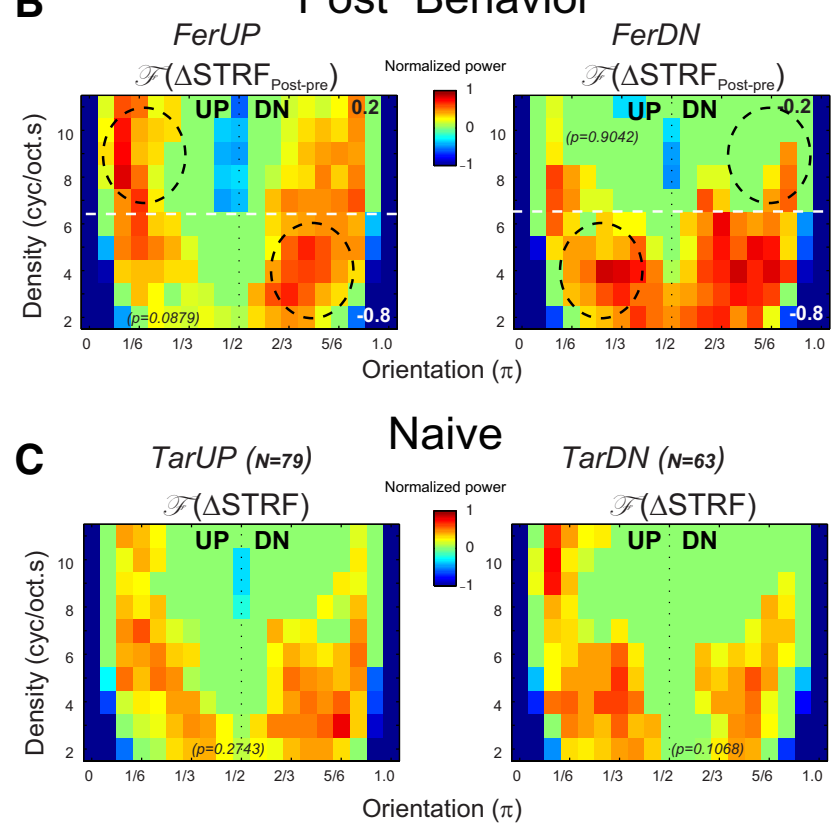

Figure 5. Population STRF changes in different behavioral states. $\boldsymbol{A}$, Averaged changes in the behaving animals. Top, Population averages of $\mathrm{F}\left(\Delta \mathrm{STRF}_{\text {Dur-pre }}\right)$ from 112 units in FerUP (left) and 65 units in FerDN (right). The average is strongly asymmetric, reflecting enhanced sensitivity to DOWN shifts in FerUP, and UP shifts in FerDN, as highlighted by the dashed circles. Note the opposite asymmetry at LD and HD regions (the white dashed lines at 7 cycles/octave.s indicate the border line that divides the two regions) in both FerUP and FerDN. The asymmetry indices $L D$ and $H D$ are computed and are given in the bottom right and top right corners of all panels. $L D$ versus $H D$ asymmetry indices from all cells are shown in the scatter plot (bottom). Consistent with the matched filter hypothesis, the points tended to lie above the midline (LD $H D<0$ ) for FerUP (paired Wilcoxon signed rank test, $p=0.0389$ ), and the opposite for FerDN (paired Wilcoxon signed rank test, $p=0.0004$ ), as confirmed by the overlaid histograms of the differences. $\boldsymbol{B}$, Averaged changes in passive post-behavior. The asymmetry of the average $F$ $\left(\triangle \mathrm{STRF}_{\text {Post-pre }}\right)$ at both $L D$ and HD region persisted in FerUP (left), but not in FerDN (right) after the behavior. C, Averaged changes in the naive animals. Responses to the same sequence of tone sequence sounds in a behaviorally naive animal demonstrate that presenting the stimuli passively induces very weak changes, with no significant asymmetry in the population average $(\mathrm{F}(\Delta \mathrm{STRF}))$.
STRF measurements, using exactly the same sequences of task stimuli described above for the trained animals, were performed in two task-naive animals (Fig. $5 \mathrm{C}$ ). These control studies were conducted to determine whether STRF changes occurred independently of behavior, and might be stimulus-driven in an untrained (nonbehaving) animal. Both versions of the tasks were simulated in each animal, i.e., with target (reference) tone sequence shifting UP (DN) and vice versa. Using exactly the same analysis procedures described above, the averaged $\mathrm{F}(\triangle \mathrm{STRF})$ values measured from 79 and 63 units in each condition exhibited no significant asymmetry, and the overall changes of the $\mathrm{F}$ ( $\triangle \mathrm{STRF}$ ) were weaker (Fig. $5 C$ ) compared with the changes in the neuronal population in the trained animals in active task versus passive conditions (Fig. 5A).

In summary, the averaged responses of a large population of A1 neurons from ferrets trained on a pitch-contour discrimination task exhibited rapid plasticity of their STRFs during behavior. The change enhanced the spectrotemporal representation of the direction of tone sequence shifts presented during the reference epoch of the trials, which were the stimuli associated with a negative reward (i.e., timeout for licking during the epoch of reference stimuli). Furthermore, the specific and predicted pattern of spectrotemporal plasticity was significant only in the trained animals and was absent in two naive animals. These findings are consistent with the "matched-filter hypothesis" that previously was shown to successfully predict plasticity during purely spectral tasks.

\section{Directionality of neuronal responses in primary auditory cortex}

Earlier studies have generally found that auditory cortical responses to a tone in a sequence largely depended on the sequence context and hence were not predictable from responses to the same tones in a different sequence context or when presented in isolation. A1 neurons have been shown to exhibit forward suppression (Brosch and Schreiner, 1997, 2000; Brosch et al., 1998) and also intrinsic facilitation (Brosch and Schreiner, 2000; Brosch et al., 1999) to two-tone sequences in a "masker-probe" configuration. Thus, sequence-sensitive neurons are quite common in A1 and may play an important role in the cortical representation of spectrotemporal patterns of acoustic signals.

In a "masker-probe" configuration, the first tone (T1, the masker) varied in frequency and intensity, whereas the second tone (T2, the probe) was fixed and placed at the neuron's characteristic frequency (Brosch and Schreiner, 1997, 2000; Brosch et al., 1998, 1999). In the present studies, the 81 two-tone sequences were randomly combined from 9 frequencies around the neuron's BF (see Materials and Methods); hence, both T1 and T2 varied in frequency. We found the effects of $\mathrm{T} 1$ on neuronal responses to T2 were also dependent on the spectral distance of T2 from the neuron's BF.

The complexity of the response patterns resulting from the $\mathrm{BF}$ dependence effect is illustrated in Figure 6 from two A1 neurons in the naive ferret. The two neurons had BFs at $\sim 707 \mathrm{~Hz}$ and 2000 $\mathrm{Hz}$ (Fig. $6 A$ ). In Figure $6 B$, the red (blue) raster and response poststimulus time histograms represent neuronal activity to the upward (downward) two-tone sequences and are arranged by $\mathrm{T} 2$ frequencies. A similar magnitude of neuronal responses was evoked by $\mathrm{T} 2$ when $\mathrm{T} 2$ was placed at the neuron's BF. This was observed in both upward (the T1 was one-half octave lower than $\mathrm{T} 2$ ) and downward (T1 was one-half octave higher than T2) sequences (Fig. 6B, marked panel, red asterisk). However, a larger response was found in upward tone sequence than in downward 
A

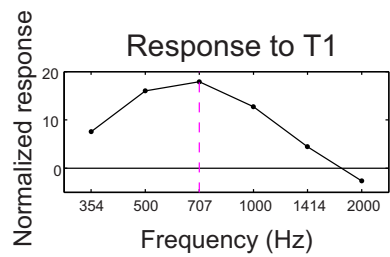

B

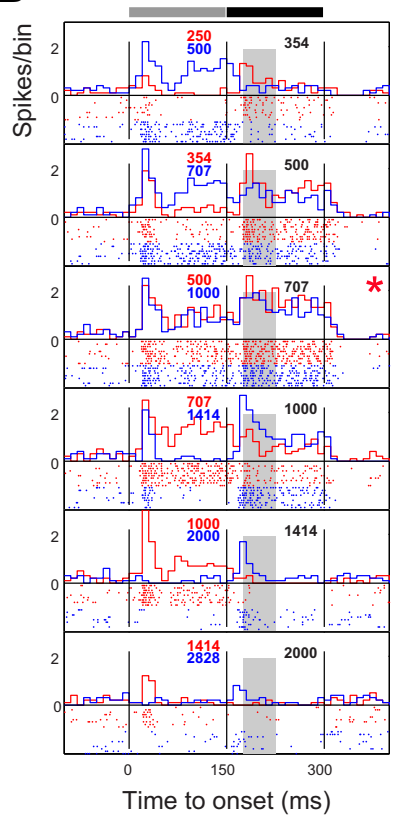

C

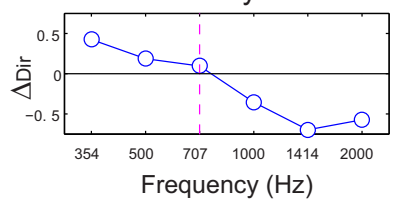

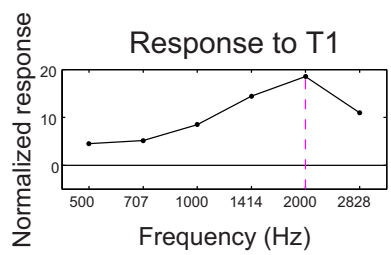

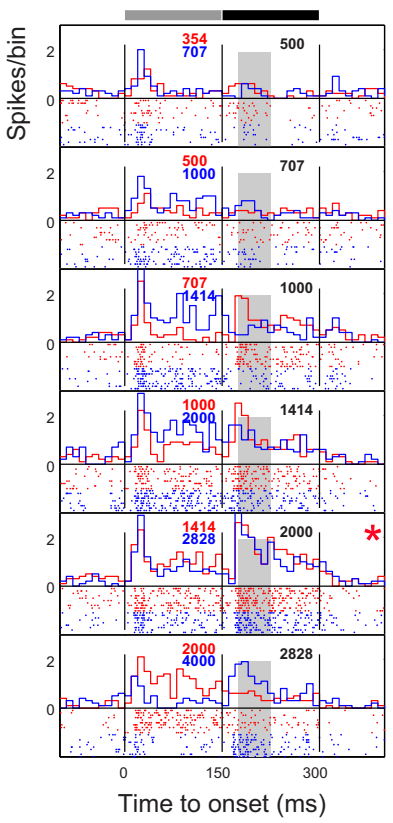

Directionality function

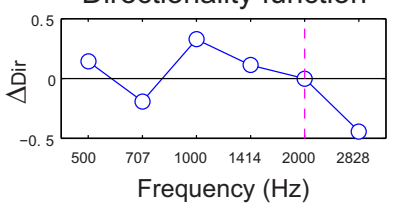

D

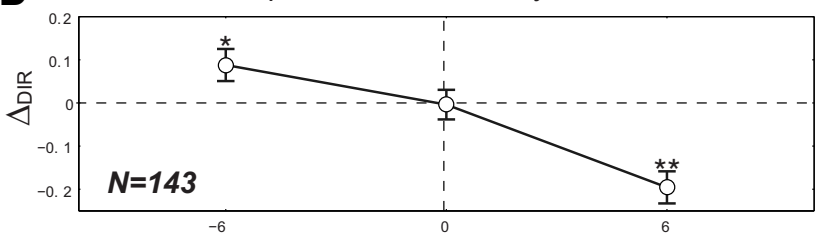

T2 from BF (st)

Figure 6. Two examples of directionality functions of A1 neurons. $\boldsymbol{A}$, Frequency response curves. Neuron's frequency response curves are computed from the onset responses at different $\mathrm{T} 1$ frequency, which are normalized to each neuron's spontaneous activity. The BF is defined as the frequency inducing the maximum responses as indicate by the dashed vertical line. $\boldsymbol{B}$, Raster and poststimulus time histogram representation of the response to the tone sequence. The responses to UP-step (the red) and DOWN-step (the blue) tone sequences are first aligned relative to the $\mathrm{T} 2$ frequency and then superimposed. The frequencies of the $\mathrm{T} 1$ (red and blue) and $\mathrm{T} 2$ tones (black) of the sequence are indicated inside each panel. ${ }^{*}$ The panel in which the T2 frequency was at the neuron's BF. The vertical line in each panel indicates the onset or offset of the T1 and T2 tones. Top, Horizontal bars represent the duration of T1 (filled with gray) and T2 (filled with black). C, Directionality function of the neurons. The directional selectivity $\left(\Delta_{\mathrm{DIR}}\right)$ of the response to the tone sequence was computed from the onset responses ( $25-75 \mathrm{~ms}$ onset time windows, shaded areas in $\boldsymbol{B}$ ) to T2 at different T2 frequencies (see Materials and Methods). Neurons are not sensitive to the step direction in the sequence when the $\mathrm{T} 2$ is at the neuron's $\mathrm{BF}$ $\left(\Delta_{\text {DIR }}\right.$ is near zero); however, they are sensitive to UP (DOWN) shift sequence when T2 frequency is below (above) the BF within the receptive field. $\boldsymbol{D}$, Population directionality functions of $\mathrm{A} 1$ neurons. The averaged directionality function from a neuronal population $(N=143)$ in $A 1$ of

sequence when the T2 frequency was placed below the neuron's BF (Fig. 6B, panels above the red asterisk), whereas a larger response was found in downward sequences than in upward sequences when the T2 frequency was above the neuron's BF (Fig. $6 B$, panels below the red asterisk).

To summarize and simplify the presentation of these results, we computed a directional selectivity index $\left(\Delta_{\mathrm{DIR}}\right)$ for all tested A1 neurons as follows: $\Delta_{\mathrm{DIR}}=\left(\mathrm{R}_{\mathrm{UP}}-\mathrm{R}_{\mathrm{DN}}\right) /\left(\mathrm{R}_{\mathrm{UP}}+\mathrm{R}_{\mathrm{DN}}\right)$, where $\mathrm{R}_{\mathrm{UP}}\left(\mathrm{R}_{\mathrm{DN}}\right)$ are $\mathrm{T} 2$ onset responses when preceded by a UP (DN) step, respectively (see Materials and Methods). By computing the $\Delta_{\text {DIR }}$ at each T2 frequency, it was possible to summarize the directionality function for each neuron as shown in Figure 6C. In general, within a neuron's receptive field, there was a stronger response to the upward tone sequence when $\mathrm{T} 2<\mathrm{BF}$, and to the downward sequence when $\mathrm{T} 2>\mathrm{BF}$. There was weak or almost no directional selectivity when the T2 frequency was near or at neuron's BFs (Fig. $6 C$, vertical dashed line).

The population average directionality function from all neurons $(N=143)$ recorded in the two naive ferrets is shown in Figure $6 D$. The population average was aligned by the distance of the T2 frequency from each neuron's BF. The population average of $\Delta_{\mathrm{DIR}}$ is significantly greater (below) than zero $(p<0.05$, sign test) when T2 frequency is one-half octave away from BF, which indicates a significant preference to upward (downward) tone sequences. However, the average $\Delta_{\text {DIR }}$ of the neuron population is $\sim 0$ when the T2 frequency is at or near the neurons' BF, indicating a lack of directional preference. This pattern of directional sensitivity (directionality function) is a common characteristic of A1 neurons when encoding the directionality of two tone sequences and hence is a useful metric for assessing the changes of neuron's directional sensitivity to tone sequences induced by training and by engagement in task performance.

\section{Evidence of directional plasticity in tone-pair responses}

We recorded responses to tone sequences while animals performed the two-tone sequence discrimination task in the absence of background TORC stimuli (Table 1, second dataset) as illustrated by the stimuli in Figure $1 A, B$ (top). Several features of the response were measured, including the onset responses to both T1 (to compute neurons' BFs) and T2 (to measure the frequency shift between the two tones). We then computed the directionality functions (following exactly the same procedures as in Fig. 6D) from FerUP and FerDN before, during, and after performance of the tasks, as well as from two naive animals, which are all illustrated in Figure 7.

Figure $7 A$ illustrates the population averaged directionality functions from trained animals (Table 1: 115 cells in FerUP; 58 cells in FerDN). As expected from the above analysis, the overall (sloping) tendency of all directionality functions reflected the effects of the tuning curves and the forward adaptive effects of T1 on the cells' response to T2 (Shamma and Symmes, 1985; Brosch and Schreiner, 1997; Brosch et al., 1998, 1999, 2004; Bartlett and Wang, 2005; Brosch and Scheich, 2008). This trend was largely independent of the behavioral state of the animal, and hence was seen in all behavioral states, e.g., in passive (black and green lines) or behavioral states (red lines).

two naive ferrets confirms a general tendency observed around the neuron's BF in the two examples in $C .{ }^{*} p<0.05$, significant bias in directional selectivity ( $t$ test). ${ }^{* *} p<0.01$, significant bias in directional selectivity ( $t$ test). 


\section{A Behaving}
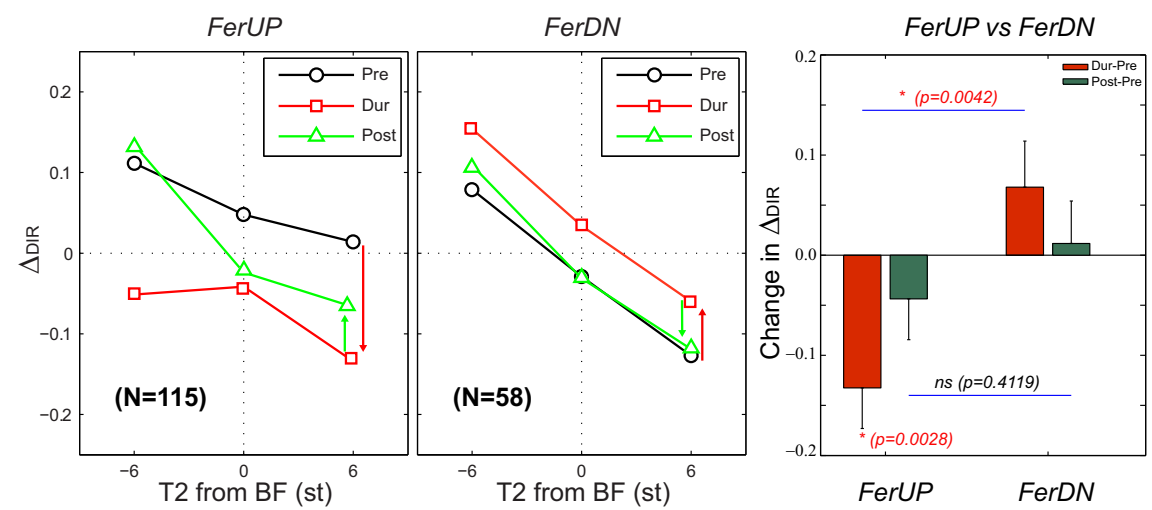

B Naive
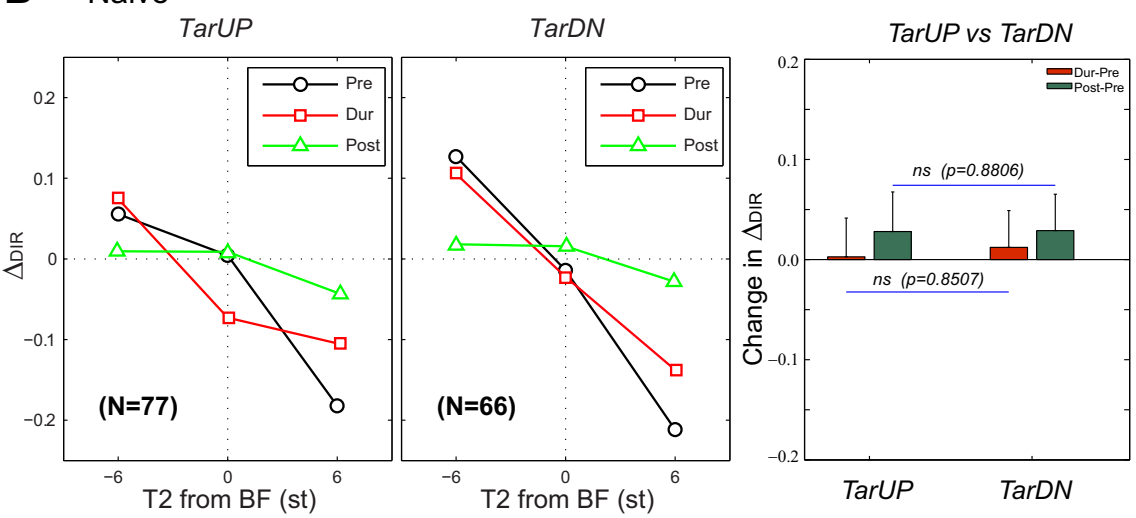

Figure 7. Evidence of spectrotemporal plasticity in directionality functions. Rapid plasticity in responses to the tone pairs led to similar conclusions as those derived from analysis of STRF changes (based on responses to TORCS). $\boldsymbol{A}$, Left, Middle, The directionality index function $\Delta_{D I R}$ (ordinate) reports the directional selectivity of unit responses to upward or downward tone-pair shifts. The index varies with the frequency of the second tone (T2) relative to the BF of the cell (abscissa) (e.g., for T2 at 6 to -6 semitones [st] relative to the $\mathrm{BF}$ of each cell). During behavior, the average indices from all FerUP cells shift significantly toward negative values (red curve and arrow), indicating an enhanced preference to DN shifts. The opposite occurs in FerDN responses (red curve and arrow). Measurements from the same cells after behavior (green curves and arrows) indicate that the enhancement weakly persists in FerUP after conclusion of the task, but not in FerDN, as indicated by the green arrows in each panel. Right, Summary of average index changes in the two behaving animals. The red bars represent significant changes during behavior relative to measurements in the prepassive state. In all, these shifts are toward negative indices in FerUP and toward positive indices in FerDN (i.e., in the same direction of enhancements as those found in the STRF changes). *Statistical significance for the change of directional index during versus before task by Wilcoxon signed rank test, or of the difference of the changes between the animals (FerUP vs FerDN) by Wilcoxon rank sum test. $\boldsymbol{B}$, Left, Middle, In the naive animal, there were no significant shifts during the three conditions. Right, The average index changes in the naive animal showed no significant changes in the indices across the population.

However, in both trained animals, there was a significant overall up or down translation in directionality functions during behavior compared with pre-behavior passive listening (Fig. 7A, two left panels, red vs black). This shift implies an enhanced sensitivity to the direction of the reference tone sequences, i.e., DN (or UP) steps in FerUP (or FerDN) respectively, as highlighted by Figure 7A (left and middle panels, red arrows). These enhancements were consistent with the STRF changes discussed earlier in Figure $5 A$, which were measured in the same behavioral contexts but in a different population of cells, and using very different stimuli (TORCs) and response measures ( $\mathrm{F}(\Delta \mathrm{STRF})$ ). A summary of $\Delta_{\mathrm{DIR}}$ changes during behavior from all cells in the two animals is depicted by Figure $7 \mathrm{~A}$ (right, red bars). The red bars stand significantly above or below the midline in opposite directions for the two animals (Wilcoxon rank sum test, $p<0.0042$ ), indicating enhanced sensitivity to the direction of pitch change in reference tone sequence.

The persistence of the plasticity was not significant in either animal in these measurements. In one animal (FerDN), the post-task directionality function (green curves) returned to the pretask level (the black curve) as shown in Figure $7 \mathrm{~A}$ (middle). There was, however, a weak persistent trend in the other animal (FerUP) (Fig. 7A), which is interesting in that it mirrored the pattern of persistence seen in the STRF changes (Fig. 5B), demonstrating again the correspondence between the conclusions drawn from responses of different populations of neurons, with two different types of stimuli (TORCs vs tone sequences), and distinct response measures (STRFs vs $\Delta_{\text {DIR }}$ ).

In contrast to the behaving animals, naive passively listening animals did not show any significant patterns of change in the directionality functions when measured with exactly the same task stimuli (Fig. 7B). The results again replicate the earlier findings derived from STRF measurements with TORCs in the same naive animals (Fig. 5C).

\section{Overall plasticity of response parameters and receptive field shapes}

To explore further whether there were other task-related changes in receptive field properties, we measured several other response and receptive field shape parameters from the STRFs obtained before, during, and after behavior, including unit BF, peak response power, spectral and temporal asymmetry, response duration, peak response latency, and separability (Shechter et al., 2007; Simon et al., 2007). All these measured receptive field parameters exhibited negligible or inconsistent changes during behavior in both animals, except a decrease in the proportion of separable units' STRFs (Shechter et al., 2007), defined in the Fourier transform domain as in Simon et al. (2007). A fully separable STRF has a symmetric F (STRF). Therefore, as expected from the results shown in Figures 4 and 5 , changes during behavior altered the asymmetry of the STRFs, and hence their separability.

\section{Discussion}

Animals were trained to discriminate variable frequency tone pairs based on the direction of pitch change in two-tone sequences (i.e., based on the two-tone contours regardless of their absolute frequencies) (Yin et al., 2010). In such an auditory cognitive task, the acoustic features critical for task performance were jointly spectrotemporal. The findings we describe here point to two basic conclusions: (1) rapid plasticity during task performance occurs in a broader spectrotemporal domain (Fritz et al., 2003); and (2) the valence of this plasticity is strictly deter- 
mined by the aversive or appetitive meaning of the task-stimuli, consistent with earlier results (Fritz et al., 2005a; David et al., 2012).

These results were confirmed in each of two trained animals performing the same task, but with different reward associations, and from responses in two independent populations of cells in each trained animal, measured with completely different stimuli, and with diverse response measures. In many of our previous studies (Fritz et al., 2003, 2005a, b, 2007; Atiani et al., 2009; David et al., 2012), STRF changes were used to measure task-related plasticity in A1. By contrast, direct changes of responses to task stimuli were only used where STRFs were impossible to compute because of lack of phase-locked responses to TORCs (e.g., in the prefrontal cortex) (Fritz et al., 2010). In the present study, both distinct types of measurements were used in A1, demonstrating that rapid plasticity is manifested in a variety of response measures as long as they can be extracted from the noisy variability of the responses during behavior.

\section{The matched-filter hypothesis}

Engagement in a behavioral task could induce rapid adaptive changes in receptive fields and responses of auditory cortical cells in a manner that altered the representation of the stimuli, consistent with a relative enhancement of task-relevant foreground stimuli (Fritz et al., 2003). Our earlier studies have focused on receptive field transformations in the spectral domain during tasks involving detection of spectral changes in task stimuli (Fritz et al., 2003, 2005a, b, 2007; Atiani et al., 2009; David et al., 2012). The findings have demonstrated that receptive field plasticity reflected these spectral changes, leading to the formulation of the "matched-filter hypothesis" (Fritz et al., 2007; Mesgarani et al., 2010; Lee and Middlebrooks, 2011). In contrast, this study tested the broadest implication of this hypothesis, exploring whether rapid task-related receptive field plasticity might also reflect the full spectrotemporal details of task stimuli, thus providing evidence of a more complete and richer role for behavior-induced plasticity that transcends the purely spectral dimension examined earlier.

Although our findings confirm that rapid plasticity matched the difference between the spectrotemporal representations of the two discriminated stimuli, visualizing the changes was a challenge because the animal had to extract the pitch contours from a sequence of variable frequency tone-pair stimuli. This required transformation of the STRFs and the stimuli to a common domain that is more stable with respect to changes in their parameters, such as the Fourier transform. Similar challenges to measuring rapid plasticity were encountered in tasks that used complex stimuli, such as speech (Mesgarani et al., 2009; Mesgarani and Chang, 2012). In those studies, rapid plasticity was demonstrated using an alternative approach, by reconstructing the stimulus spectrograms from the adapted responses so as to visualize more easily the changes that plasticity caused.

Rapid spectrotemporal plasticity here arose in animals performing tasks with simple spectrotemporal stimuli. There is no reason, however, why such plasticity should not also occur with more complex stimuli and tasks. Thus, we conjecture that any combination of acoustic cues that can be discriminated will induce matched rapid adaptive changes. For instance, if a task involves discriminating spatial (or binaural) cues, the rapid plasticity would take place along spatially selective dimensions of the neural response. And if the cues are also temporal (e.g., discriminating tones that move from left to right or vice-versa), the plasticity may take the form of spatiotemporal changes. We also believe that exactly the same neuronal mechanisms of rapid plasticity come into play in a complex acoustic landscape, such as the "cocktail party" setting, where we conjecture that the attended alternating foci (e.g., a pair of interacting voices against a complex acoustic background) would lead to selective spatiospectrotemporal plasticity changes that enhance perception (Mesgarani and Chang, 2012).

\section{Directional selectivity of primary auditory neurons to tone sequences}

The directional selectivity of auditory responses and their topographic distribution in primary auditory cortex (Suga, 1968; Heil et al., 1992; Zhang et al., 2003) and subcortical structures (Gittelman et al., 2009; Kuo and Wu, 2012) has been extensively studied in numerous animal species, most often using frequencymodulated (FM) tones. The emergence of this sensitivity has been attributed primarily to a temporal imbalance between inhibitory and excitatory inputs from neighboring frequencies that leads to a systematic preference for one direction of sequential activation over another (Suga, 1968; Heil et al., 1992; Zhang et al., 2003; Gittelman et al., 2009; Kuo and Wu, 2012). Similar mechanisms may explain the directional sensitivity of our tone-pair stimuli, in that they can be viewed as discontinuous approximations of the FM sweeps.

As described in detail in the results of Fig. 6, the apparent directional selectivity of a cortical response substantially depends on the parameters of the stimulus in relation to the tuning curve and dynamics of the neuron. For example, with FM sweeps, critical stimulus parameters include the starting and ending frequencies of the sweep relative to the BF of the cell, the sweep rate, and the overall level of the FM tone. Similarly, for the tone pairs, directional selectivity changes depending on T1 and T2 frequencies relative to the BF and the size of the relative shift. In short, directional selectivity reflects the spectral and temporal contexts of the tones in relation to the shape of the frequency-receptive field (Brosch and Schreiner, 1997, 2000; Brosch et al., 1998, 1999; Klampfl et al., 2012). For example, a commonly invoked aspect of the frequency-receptive field that plays a critical role in directional selectivity is the asymmetry of its central excitatory field and inhibitory surrounds (Suga, 1968; Heil et al., 1992; Brosch et al., 2000), and the pattern of their activations by tones.

Because of this complexity, we have opted to define and use the directionality function (Fig. 6) to stabilize the characterization of directional selectivity across a diverse population of neurons and a highly variable set of stimuli, and to allow us to combine physiological data from all neurons regardless of their tuning properties. During behavior, the directionality function maintained its basic sloping shape (Fig. 7), except for an up or down translation (Fig. $7 A$ ), reflecting the rapid adaptive change in favor of sensitizing responses to the direction of the aversive tone pair. The simplest mechanism to explain this translation is a change in the asymmetry of the sideband inhibition of the frequency-receptive field, making it more effective for T1 to facilitate or suppress the responses to T2 (Brosch et al., 2000). Our results are also broadly consistent with earlier findings of significant long-term tone sequence-sensitive enhancements in monkeys trained to perform a contour-direction discrimination task (Brosch et al., 2004; Selezneva et al., 2006). Auditory cortical neurons (in $\mathrm{A} 1$ and $\mathrm{CM}$ ) of monkeys trained to respond to reward-predicting downward frequency steps showed increased phasic responses only to downward steps and not to upward steps or to iso-frequency tone sequences (Selezneva et al., 2006). This preference for downward frequency steps was not observed in 
naive monkeys and appears to have been the result of a permanent change in the tuning of auditory cortical neurons (Selezneva et al., 2006). However, our results support a more nuanced interpretation, in which directional sensitivity of A1 neurons in the ferret to a two-tone contour change is highly dependent upon the location of the tone-pair frequency shift relative to the neuron's $\mathrm{BF}$ and thus it is difficult to say whether is a given neuron is an UP or a DOWN cell. Our results are based on rapid changes in sensitivity of the STRFs towards greater UP or DOWN sensitivity during task performance. The same directional changes during the task can occur in cells that initially prefer UP ripples or cells that prefer DOWN ripples. The results of Selezneva et al. (2006) reflect long-term changes in A1 following long-term training. In contrast, the results described in our study involve short-term changes in neuronal tuning during only one session of task performance. Our studies of rapid plasticity were conducted in taskproficient animals, long after their task training was completed. Although long-term changes may have occurred during training of the ferrets on the contour direction task, we did not analyze them in this study.

Our results are also in accord with earlier observations of long-term tone sequence-sensitive enhancements in monkey auditory cortex after training on a four-note melody detection task (Yin et al., 2008). Similar findings of long-term tone sequence modulation were observed in the auditory cortex of rats that were entrained by associative pairing of a three-element sequence with stimulation in nucleus basalis (Kilgard and Merzenich, 2002). The ferrets in our study may have experienced long-term changes in tuning; however, our primary focus in this study has been on rapid task-related changes during performance of the contour task. Although earlier work has shown these long-term changes, rapid neurophysiological plasticity has also been seen in human auditory cortex after only 6 min of tone-sequence discrimination learning (Gottselig et al., 2004).

\section{Valence of the rapid plasticity}

Rapid plasticity is driven by perceptual discrimination between two categories of stimuli (referred to as reference and target) and is hypothesized to enhance the neural representation of stimulus differences, and the contrast between foreground and background stimuli so as to improve perceptual performance (Fritz et al., 2007; Mesgarani et al., 2010). Although it is theoretically equally effective to achieve this goal by enhancing either stimulus and suppressing the other, we have found previously in "tone detection" and "two-tone discrimination" Go/NoGo tasks (Fritz et al., 2005a, b; David et al., 2012) that plasticity enhanced the "aversive" stimulus associated with avoidance, and suppressed the "appetitive" stimulus associated with the reward (or approach). The behavioral task described in the current study had more complex stimulus cues than in previous studies, but this basic pattern of plasticity was successfully predicted and confirmed our earlier findings. We believe it likely that this pattern will generalize to other task structures, such as the Go-NoGo delayed match-to-sample task in which the animal withholds response through a sequence of ("aversive") stimuli until a matched ("appetitive") target is received. It is an open question as to whether or how this pattern of plasticity would apply to other task designs (e.g., two-alternative forced choice) and demands (e.g., spatial discrimination tasks, or extraction of higher order auditory features, e.g., voice recognition).

In conclusion, we postulate that rapid plasticity can in principle reflect the behaviorally relevant cues in any task stimuli, which include spectrally complex and temporally extended sig- nals, such as speech syllables, words, or musical phrases. In such a scenario, rapid receptive field plasticity would function as an adaptive mechanism to quickly discriminate and retain sensory information that could serve as contextual information for subsequent processing. Such adaptations can be subsequently reinforced and stored as sensory filters or templates in longer-term memory upon further repeated exposure (Kuhl, 2004).

\section{References}

Atiani S, Elhilali M, David SV, Fritz JB, Shamma SA (2009) Task difficulty and performance induce diverse adaptive patterns in gain and shape of primary auditory receptive fields. Neuron 61:467-480. CrossRef Medline

Bao S, Chang EF, Woods J, Merzenich MM (2004) Temporal plasticity in the primary auditory cortex induced by operant perceptual learning. Nat Neurosci 7:974-981. CrossRef Medline

Bartlett EL, Wang X (2005) Long-lasting modulation by stimulus context in primate auditory cortex. J Neurophysiol 94:83-104. CrossRef Medline

Beitel RE, Schreiner CE, Cheung SW, Wang X, Merzenich MM (2003) Reward-dependent plasticity in the primary auditory cortex of adult monkeys trained to discriminate temporally modulated signals. Proc Natl Acad Sci U S A 100:11070-11075. CrossRef Medline

Blake DT, Heiser MA, Caywood M, Merzenich MM (2006) Experiencedependent adult cortical plasticity requires cognitive association between sensation and reward. Neuron 52:371-381. CrossRef Medline

Brosch M, Scheich H (2008) Tone sequence analysis in the auditory cortex of awake macaque monkeys. Exp Brain Res 184:349-361. CrossRef Medline

Brosch M, Schreiner CE (1997) Time course of forward masking tuning curves in cat primary auditory cortex. J Neurophysiol 77:923-943. Medline

Brosch M, Schreiner CE (2000) Sequence sensitivity of neurons in cat primary auditory cortex. Cereb Cortex 10:1155-1167. CrossRef Medline

Brosch M, Schulz A, Scheich H (1998) Neuronal mechanisms of auditory backward recognition masking in macaque auditory cortex. Neuroreport 9:2551-2555. CrossRef Medline

Brosch M, Schulz A, Scheich H (1999) Processing of sound sequences in macaque auditory cortex: response enhancement. J Neurophysiol 82: 1542-1559. Medline

Brosch M, Selezneva E, Bucks C, Scheich H (2004) Macaque monkeys discriminate pitch relationships. Cognition 91:259-272. CrossRef Medline

Brown M, Irvine DR, Park VN (2004) Perceptual learning on an auditory frequency discrimination task by cats: association with changes in primary auditory cortex. Cereb Cortex 14:952-965. CrossRef Medline

David SV, Hayden BY, Gallant JL (2006) Spectral receptive field properties explain shape selectivity in area V4. J Neurophysiol 96:3492-3505. CrossRef Medline

David SV, Fritz JB, Shamma SA (2012) Stimulus valence and task-relevance control rapid plasticity in primary auditory cortex. Proc Natl Acad Sci U S A 109:2144-2149. CrossRef Medline

Depireux DA, Simon JZ, Klein DJ, Shamma SA (2001) Spectro-temporal response field characterization with dynamic ripples in ferret primary auditory cortex. J Neurophysiol 85:1220-1234. Medline

Dong C, Qin L, Liu Y, Zhang X, Sato Y (2011) Neural responses in the primary auditory cortex of freely behaving cats while discriminating fast and slow click-trains. PLoS One. 6:e25895. CrossRef Medline

Fritz J, Shamma S, Elhilali M, Klein D (2003) Rapid task-dependent plasticity of spectrotemporal receptive fields in primary auditory cortex. Nat Neurosci 6:1216-1223. CrossRef Medline

Fritz J, Shamma S, Elhiliali M (2005a) Active listening: task-dependent plasticity of receptive fields in primary auditory cortex. Hearing Res 206:159 176. CrossRef Medline

Fritz JB, Elhilali M, Shamma S (2005b) Differential dynamic plasticity of A1 receptive fields during multiple spectral tasks. J Neurosci 25:7623-7635. CrossRef Medline

Fritz JB, Elhilali M, Shamma SA (2007) Adaptive changes in cortical receptive fields induced by attention to complex sounds. J Neurophysiol 98: 2337-2346. CrossRef Medline

Fritz JB, David SV, Radtke-Schuller S, Yin P, Shamma SA (2010) Adaptive, behaviorally-gated, persistent encoding of task-relevant auditory information in ferret frontal cortex. Nat Neurosci 13:1011-1019. CrossRef Medline 
Gittelman JX, Li N, Pollak GD (2009) Mechanisms underlying directional selectivity for frequency-modulated sweeps in the inferior colliculus revealed by in vivo whole-cell recordings. J Neurosci 29:13030-13041. CrossRef Medline

Gottselig JM, Brandeis D, Hofer-Tinguely G, Borbély AA, Achermann P (2004) Human central auditory plasticity associated with tone sequence learning. Learn Mem 11:162-171. CrossRef Medline

Guo F, Intskirveli I, Blake DT, Metherate R (2013) Tone-detection training enhances spectral integration mediated by intracortical pathways in primary auditory cortex. Neurobiol. Learn Mem 101:75-84. CrossRef Medline

Heil P, Langner G, Scheich H (1992) Processing of frequency-modulated stimuli in the chick auditory analogue: evidence of topographic representations and possible mechanisms of rate and directional selectivity. J Comp Physiol A 171:583-600. Medline

Kilgard MP, Merzenich MM (2002) Order sensitive plasticity in adult primary auditory cortex. Proc Natl Acad Sci U S A 99:3205-3209. CrossRef Medline

Klampfl S, David SV, Yin P, Shamma SA, Maass W (2012) A quantitative analysis of information about past and present stimuli encoded by spikes of A1 neurons. J Neurophysiol 108:1366-1380. CrossRef Medline

Klein D, Simon J, Depireux D, Shamma S (2000) Robust spectro-temporal reverse-correlation for the auditory system: optimal stimulus design. J Comp Neurosci 9:85-111. CrossRef Medline

Kowalski N, Depireux DA, Shamma SA (1996) Analysis of dynamic spectra in ferret primary auditory cortex: I. Characteristics of single-unit responses to moving ripple spectra. J Neurophysiol 76:3503-3523. Medline

Kuhl PK (2004) Early language acquisition: cracking the speech code. Nat Rev 5:831-843. CrossRef Medline

Kuo RI, Wu GK (2012) The generation of direction selectivity in the auditory system. Neuron 73:1016-1027. CrossRef Medline

Lee CC, Middlebrooks JC (2010) Auditory cortex spatial sensitivity sharpens during task performance. Nat Neurosci 14:108-114. CrossRef Medline

Lu T, Liang L, Wang X (2001) Neural representation of temporally asymmetric stimuli in the auditory cortex of awake primates. J Neurophysiol 85:2364-2380. Medline

McKenna TM, Weinberger NM, Diamond DM (1989) Responses of single auditory cortical neurons to tone sequences. Brain Res 481:142-153. CrossRef Medline

Mesgarani N, Chang EF (2012) Selective cortical representation of attended speaker in multi-talker speech perception. Nature 485:233-236. CrossRef Medline

Mesgarani N, David SV, Fritz JB, Shamma SA (2009) Influence of context and behavior on the population code in primary auditory cortex. J Neurophysiol 102:3329-3339. CrossRef Medline
Mesgarani N, Fritz J, Shamma SA (2010) A computational model of rapid task-related plasticity of auditory cortical receptive fields. J Comp Neurosci 28:19-27. CrossRef Medline

Miller LM, Escabí MA, Read HL, Schreiner CE (2002) Spectrotemporal receptive fields in the lemniscal auditory thalamus and cortex. J Neurophysiol 87:516-527. Medline

Niwa M, Johnson JS, O'Connor KN, Sutter ML (2012) Active engagement improves primary auditory cortical neurons' ability to discriminate temporal modulation. J Neurosci 32:9323-9334. CrossRef Medline

Patil K, Pressnitzer D, Shamma S, Elhilali M (2012) Music in our ears: the biological bases of musical timbre perception. PLoS Comput Biol 8:e1002759. CrossRef Medline

Polley DB, Steinberg EE, Merzenich MM (2006) Perceptual learning directs auditory cortical map reorganization through top-down influences. J Neurosci 26:4970-4982. CrossRef Medline

Selezneva E, Scheich H, Brosch M (2006) Dual time scale for categorical decision making in auditory cortex. Curr Biol 16:2428-2433. CrossRef Medline

Shamma S, Symmes D (1985) Patterns of inhibition in the responses of auditory cortical neurons in the squirrel monkey. Hearing Res 19:1-13. CrossRef Medline

Shechter B, Depireux DA (2007) Stability of spectro-temporal tuning over several seconds in primary auditory cortex of the awake ferret. Neuroscience 148:806-814. CrossRef Medline

Shetake JA, Engineer ND, Vrana WA, Wolf JT, Kilgard MP (2012) Pairing tone trains with vagus nerve stimulation induces temporal plasticity in auditory cortex. Exp Neurol 233:342-349. CrossRef Medline

Simon JZ, Depireux DA, Klein DJ, Fritz JB, Shamma S (2007) Temporal symmetry in primary auditory cortex: implications for cortical connectivity. Neural Comput 19:583-638. CrossRef Medline

Suga N (1968) Analysis of frequency modulated and complex sounds by single auditory neurons in bats. J Physiol 198:51-80. Medline

van Wassenhove V, Nagarajan SS (2007) Auditory cortical plasticity in learning to discriminate modulation rate. J Neurosci 27:2663-2672. CrossRef Medline

Wehr M, Zador AM (2005) Synaptic mechanisms of forward suppression in rat auditory cortex. Neuron 47:437-445. CrossRef Medline

Yin P, Mishkin M, Sutter M, Fritz JB (2008) Early stages of melody processing: stimulus-sequence and task-dependent neuronal activity in monkey auditory cortical field Al and R. J Neurophysiol 100:3009-3029. CrossRef Medline

Yin P, Fritz JB, Shamma SA (2010) Do ferrets perceive relative pitch? J Acoust Soc Am 127:1673-1680. CrossRef Medline

Zhang LI, Tan AY, Schreiner CE, Merzenich MM (2003) Topography and synaptic shaping of direction selectivity in primary auditory cortex. Nature 424:201-205. CrossRef Medline 